United Nations Educational Scientific and Cultural Organization

and

International Atomic Energy Agency

THE ABDUS SALAM INTERNATIONAL CENTRE FOR THEORETICAL PHYSICS

\title{
SYNCHRONIZATION OF DIFFUSIVELY COUPLED OSCILLATORS NEAR THE HOMOCLINIC BIFURCATION
}

\author{
Dmitry Postnov \\ Department of Physics, Saratov State University, \\ Astrakhanskaja st.83, Saratov, 410026, Russian Federation \\ and \\ Department of Physics, Chungbuk National University, Cheongju, Chungbuk 360-763, Korea, \\ Seung Kee Han \\ Department of Physics, Chungbuk National University, Cheongju, Chungbuk 360-763, Korea \\ and \\ Hyungtae Kook* \\ Department of Physics, Kyungwon University, Sungnam, Kyunggi 461-701, Korea \\ and \\ The Abdus Salam International Centre for Theoretical Physics, Trieste, Italy.
}

\begin{abstract}
It has been known that a diffusive coupling between two limit cycle oscillations typically leads to the inphase synchronization and also that it is the only stable state in the weak coupling limit. Recently, however, it has been shown that the coupling of the same nature can result in the distinctive dephased synchronization when the limit cycles are close to the homoclinic bifurcation, which often occurs especially for the neuronal oscillators. In this paper we propose a simple physical model using the modified van der Pol equation, which unfolds the generic synchronization behaviors of the latter kind and in which one may readily observe changes in the sychronization behaviors between the distinctive regimes as well. The dephasing mechanism is analyzed both qualitatively and quantitatively in the weak coupling limit. A general form of coupling is introduced and the synchronization behaviors over a wide range of the coupling parameters are explored to construct the phase diagram using the bifurcation analysis.
\end{abstract}

\author{
MIRAMARE - TRIESTE \\ September 1998
}

*Regular Associate of the Abdus Salam ICTP. 


\section{INTRODUCTION}

Synchronizations between oscillations are abundant in a variety of situations ranging from physical to biological phenomena $[1,2]$. In particular, recent studies to understand information processings of the nervous systems have been guided by the idea that synchronization of oscillatory neuronal units may provide a mechanism for functioning of the neural systems, which has been supported by experimental observations [3,4]. More specifically, it has been suggested that the temporal correlation scheme among oscillatory neuronal units may underlie the mechanism for the feature binding and segmentation in the sensory perceptions [5].

A prototype of the nonlinear oscillations may be provided by the well-known van der Pol oscillator which was originally devised as a model in the electronic circuit theory [6]. Dynamic behaviors of the oscillator are rather simply predicted from the existence of a single equilibrium (source) and a single limit cycle in the phase space. Often arising in many physical systems with an inherent nonlinear energy disspation, such a limit cycle oscillation naturally occurs as a balance between the energy generation at a small amplitude oscillation near the source and the energy dissipation at a large amplitude. The coupled dynamics of such oscillations has also been studied extensively and it has been well known from the literatures that a diffusive coupling between two such oscillations typically leads to the inphase synchronization and also that the inphase synchronization is the only stable state in the weak coupling limit $[7,8]$.

Meanwhile, a rather different kind of the synchronization behaviors has been observed for the diffusively coupled neuronal oscillators $[9,10]$. The classical model for the electrophysiological activities of a neuron has been provided by Hodgkin and Huxley [11], which is given as coupled differential equations of four degrees of freedom. The model can be reduced to simpler ones while retaining some of the important features of the neuronal dynamics. The examples of the reduced models include the Morris-Lecar [12] and the Hindmarsh-Rose model [13]. Even though these models may exhibit different specifics in datails, the structure of phase space is qualitatively the same and, therefore, the dynamics display similar behaviors. Namely, the structure of phase space is based on the existence of three equilibria and a limit cycle. The three equilibria correspond to a stable node $(N)$, a saddle $(S)$, and an unstable focus $(F)$, respectively. The limit cycle is located at the boundary for flows diverging from the focus. A typical phase portrait is depicted in Fig. VI.

The two coexisting attractors represent the two possible states of a spiking neuron. That is, the stable node corresponds to the resting state (the equilibrium state) and the limit cycle to the firing state of a neuron. The stable manifolds of the saddle separate the phase space into two attraction basins. Consequently, a stimulus to a resting neuron may not lead to firing of the neuron unless it is strong enough to push the trajectory over the separatrix into the other basin for the firing state.

When two neurons are in the firing state and are coupled to each other diffusively, the synchronization behavior becomes quite different from the case of the coupled van der Pol oscillators. That is, it has been shown that for the coupled neuronal oscillators the inphase synchronization may lose 
its stability and, in particular, the dephased synchronization can occur as the only stable state in the regime of the weak coupling strength, which is contrasted with the previous observations in the coupled van der Pol oscillators [10]. In the cited studies it has been indicated that the instability of the inphase synchronization is essentially due to the vector field deformation influenced by the presence of the saddle point near the limit cycle oscillations, which implies that the dephasing may be enhanced when the limit cycle oscillation gets close to the homoclinic bifurcation situation.

In the present work we elucidate the mechanism of the dephased synchronization of the diffusively coupled oscillators and show that a distinctive synchronization behavior is provided by the coupled oscillator systems in which the limit cycles are close to the homoclinic bifurcation. In the followings, we propose as a generic model a simple physical model using the modified van der Pol equation that has the structure of the phase space depicted in Fig. VI. In the weak coupling limit the dephasing mechanism of the synchronized oscillations near the saddle is analyzed both qualitatively and quantitatively. A geral form of coupling between the oscillators is considered by introducing coupling between the both variables of the oscillators. Then, the synchronization behaviors over a wide range of the coupling parameters are explored using the techniques of the bifurcation analysis to construct the phase diagrams. The results observed from the proposed model is also compared with the specific example of the neuronal oscillator model.

\section{MODIFIED VAN DER POL OSCILLATOR}

A general form of two-dimensional oscillatory systems can be given in the following form:

$$
\ddot{x}+F_{1}(x, \dot{x}, \mathbf{p}) \dot{x}+F_{2}(x, \mathbf{p})=0
$$

where the vector $\mathbf{p}$ represents a set of the control parameters. The functions $F_{1}$ and $F_{2}$ can be given arbitrarily as long as they fulfill conditions for the existence of an oscillation. In the given form $F_{1}$ is responsible for the energy dissipation and $F_{2}$ for the force exerted on the oscillator. The zeroes of $F_{2}$ determine the locations of the equilibria, and their stabilities are determined from the signs of $d F_{2} / d x$ and $F_{1}$ at each equilibrium: $d F_{2} / d x$ is negative only for saddles and a node, or a focus, is stable when $F_{1}$ is positive.

The simplest example of a nonlinear oscillator of such form is given by the van der Pol oscillator; $F_{1}=\alpha\left(x^{2}-1\right)$ and $F_{2}=x$. For positive $\alpha$ the only equilibrium at $x=0$ is an unstable focus since $F_{1}$ is negative for $|x|<1$. In addition, since the energy is dissipated at the large distances $(|x|>1)$, there also exists a stable limit cycle enclosing the focus with a finite amplitude. Consequently, the global dynamic behavior of the van der Pol oscillator can be predicted from the simple structure of the phase space that contains only one unstable focus and a limit cycle.

The neuronal oscillator models can be often represented in the form of Eq. (1). That is, taking the Morris-Lecar model as an example, those functions are given as

$$
F_{1}(v, \dot{v})=\frac{\partial m_{\infty}(v)}{\partial v}(v-1)+\frac{f}{\tau_{w}(v)}
$$




$$
\begin{aligned}
& +\frac{m_{\infty}\left(1-v_{k}\right)+\bar{g}_{L}\left(v-v_{L}\right)+I_{d c}-\dot{v}}{v-v_{k}}, \\
F_{2}(v)= & \frac{f}{\tau_{w}(v)}\left\{\bar{g}_{K}\left(v-v_{K}\right) w_{\infty}(v)\right. \\
& \left.+m_{\infty}(v)(v-1)+\bar{g}_{L}\left(v-v_{L}\right)-I_{d c}\right\},
\end{aligned}
$$

where the variable $v$ stands for the membrane potential of the neuron and $I_{d c}$ is the external current input which plays as a main control parameter of the model. More details for the model equation with the notations for the other parameters can be easily found in the literatures [10,12].

In the typical regime of the parameters, $F_{2}$ has three zeroes that correspond to a stable node, a saddle, and a focus, respectively. Also, when the stable and the unstable manifold of the saddle are connected via the homoclinic bifurcation, a limit cycle is born that typically encloses the unstable focus. Therefore, as it takes place, the homoclinic bifurcation separates the regime of the sustained firing (limit cycle oscillation) of the neuron, as will be described below with the Morris-Lecar model. The existence of the homoclinic bifurcation appears as a common feature of the neuronal models.

Going back to the Morris-Lecar model example, for a small external current $I_{d c}$ the only stable state is the stable node that corresponds to the resting state of a neuron; typically, the focus is unstable near the homoclinic bifurcation. Nearby the stable node a saddle exists whose stable manifolds separates the response of a resting neuron against a stimulus into two kinds; this is often referred to as the 'all-ornone' firing behavior of a neuron. That is, a small stimulus does not induce firing, which corresponds to a short excursion of the phase flow being attracted eventually to the stable node, whereas a large stimulus over a threshold level may lead to firing of the neuron, which corresponds a long excursion of the flow across the separatrix. For the latter case the flow is also eventually attracted to the stable node where the neuron completes one firing. The firing is not sustained at this regime unless the stimulus is repeated.

However, as $I_{d c}$ is increased, the homoclinic bifurcation occurs at $I_{d c} \approx 0.0729$ on which the stable and the unstable manifold of the saddle are connected to form a loop homoclinic to the saddle. Beyond the bifurcation point, a stable limit cycle occurs, the flow on which corresponds to the sustained periodic firings. Consequently, in this parameter regime of the model, the phase space contains three equilibria, a stable node, a saddle and an unstable focus, and a limit cyle, as its typical phase protrait can be also given by Fig. VI. This structure of the phase space can be readily predicted from the shape of the functions $F_{1}$ and $F_{2}$ as shown in Fig. VI(a). That is, three equilibria are located at the zeroes of $F_{2}$ and the type of each equilibrium is determined from the signs of $F_{1}$ and $d F_{2} / d x$. In the figure, $F_{1}$ is given as a contour plot in the $(v, \dot{v})$ plane and the dark area corresponds to the negative dissipation (the energy generation).

As indicated above the limit cycle oscillations near the homoclinic bifurcation are typical in the neuronal oscillators. Therefore, in understanding systematically the generic behaviors of the coupled dynamics between such oscillations it would be desirable to have a model that is easily controllable near the bifurcation and shares the features of interest with the neuronal oscillators as well. For this purpose we propose a simple model as follows and the present work will be focussed on the quantitative 
descriptions for this model.

The model is obtained from the van der Pol oscillator, while a slight modification is needed to maintain the required structure of the phase space; hereafter, this model will be called as the modified van der Pol (MVP) model. That is, to have three equilibria, we need to introduce a nonlinear cubic force:

$$
\begin{aligned}
& F_{1}(x)=\alpha\left(x^{2}-\mu\right), \\
& F_{2}(x)=x(x+d)(x+2 d) / d^{2},
\end{aligned}
$$

where $\alpha, \mu$ and $d$ are the control parameters that assume positive values.

The MVP model maintains the features of the neuronal models in that the phase space has basically the same structure as shown in Fig. VI. The function plots for $F_{1}$ and $F_{2}$ are shown in Fig. VI(b). The three equilibrium points are located at $\dot{x}=0$ and $x_{F, S, N}=0,-d,-2 d$ for the focus, the saddle, and the stable node, respectively, and the slopes of $F_{2}$ at the equilibria are $d F_{2} / d x=2,-1,2$, respectively. The focus is unstable since $F_{1}$ is negative at $x_{F}$ and the limit cycle is located in between the unstable focus and the saddle. In this presentation we set $d=3$ and $\alpha=0.2$. For the fixed values of $d$ and $\alpha$, the distance to the homoclinic bifurcation is controlled by $\mu$; the limit cycle gets closer to the saddle as $\mu$ is increased and the homoclinic connection occurs at $\mu \approx 1.255$. The limit cycle far from the bifurcation with a small $\mu$ reduces to the similar situation to the van der Pol oscillation.

In the following sections we will consider various coupling configurations. For this purpose it turns out that a more convenient form for the MVP model is provided by the canonical form of Eq. (1):

$$
\begin{aligned}
& \dot{x}=y, \\
& \dot{y}=-F_{1}(x) y-F_{2}(x) .
\end{aligned}
$$

\section{DEPHASING OF SYNCHRONIZED OSCILLATIONS NEAR THE SADDLE}

In this section, using the coupled MVP model, we consider the synchronization behavior between two limit cycle oscillations near the homoclinic bifurcation based on the dephasing mechanism of phase flows near the saddle point.

Let us consider a simpler case of the single-variable and we also assume that the coupling is sufficiently weak so that the perturbation raised in each subsystem is negligibly small. That is, the coupled MVP model with a diffusive position-variable coupling is given as

$$
\begin{aligned}
& \dot{x_{1}}=y_{1}+\varepsilon\left(x_{2}-x_{1}\right), \\
& \dot{y_{1}}=-F_{1} y_{1}-F_{2},
\end{aligned}
$$

where $\varepsilon$ is assumed to be sufficiently small. The coupling term for the other oscillator is given symmetrically as $\varepsilon\left(x_{1}-x_{2}\right)$. 
Fig. VI shows the contour plot for the magnitude of the phase velocity $v_{\phi}$ for the single oscillator in the absence of coupling; the phase velocity defined as $v_{\phi}=\sqrt{\dot{x}^{2}+\dot{y}^{2}}$ vanishes at the equilibria $(S$ and $F)$. For small $\mu$ values, the limit cycles are located close to the focus. Then the phase space structure in terms of the $v_{\phi}$-surface along $\Gamma_{1}$ is qualitatively equivalent to that of the van der Pol oscillation for which it is known that a diffusive coupling typically leads to the stable inphase synchronization. An examplary limit cycle trajectory at $\mu=0.2$ is depicted as $\Gamma_{1}$ in the figure.

However, for increased $\mu$, the limit cycle gradually approaches close to the saddle and then the shape of the $v_{\phi}$-surface explored by the limit cycle becomes qualitatively different from the small $\mu$ case. The limit cycle trajectory at $\mu=1.0$ is plotted and labelled as $\Gamma_{2}$ in Fig. VI. A trajectory on $\Gamma_{2}$ spends most time near the saddle and, therefore, the interaction due to coupling in this region becomes important. Notice that since the vector field and also $v_{\phi}$ change as $\mu$ changes it is impossible to plot both $\Gamma_{1}$ and $\Gamma_{2}$ in the same plot. Accordingly, in Fig. VI, $\Gamma_{2}$ has been plotted with a numerical accuracy while the plot of $\Gamma_{1}$ has been added only schematically to help compare the two cases.

The flows of the vector field in the vicinity of the saddle and the dephasing behavior in the presence of coupling are depicted schematically in Fig. VI for the case of the diffusive position-coupling as given in Eq. (5). The circles around $S$ represent the $v_{\phi}$ contour lines and $W^{s}$ and $W^{u}$ represent the stable and the unstable manifolds of the saddle, respectively. $\Gamma$ denotes the limit cycle trajectory in the absence of coupling and $\Gamma_{s}$ and $\Gamma_{f}$ denote trajectories perturbed due to coupling, as will be explained further below.

Suppose there is a small time lag between two oscillators when they enter into the vicinity of the saddle. That is, one oscillator $\left(\Omega_{1}\right)$ advances in phase the other $\left(\Omega_{2}\right)$ as denoted at the bottom of $\Gamma$. Then, the coupling force on $\Omega_{1}$ acts in the positive $x$ direction where the flow velocity is faster, whereas the coupling force on $\Omega_{2}$ acts in the other direction where the flow velocity is slower. Therefore, the trajectory of $\Omega_{1}$ is gradually pushed to the trajectory $\Gamma_{f}$ which is faster than $\Gamma$ and, the trajectory of $\Omega_{2}$ to $\Gamma_{s}$ which is slower. As a result, the initial time lag diverges. This implies that the inphase synchronization can be unstable, which is contrary to the synchronization behavior observed in the coupled van der Pol oscillators.

It should be notified that the dephasing does not necessarily occur all the time when the limit cycle is close to the saddle point. A similar consideration can be also given to show that the diffusive 'velocity-coupling' leads to the inphase synchronization; in this case the coupling force would be in the vertical direction in Fig. VI, and hence attractive.

To get a quantitative estimate for the dephasing between the oscillators, let us first divide the limit cycle into two pieces by a line $A B$ as shown in Fig. VI. It is expected that the effect of coupling in the region of the saddle (to the left of $A B$ ) is much more relevant to the synchronization. We introduce the measures $P$ and $Q$ for the linear rate of dephasing as follows.

$$
\begin{aligned}
& \Delta t(B)=P \Delta t(A), \\
& \Delta t(A)=Q \Delta t(B),
\end{aligned}
$$


where $\Delta t$ in the right-hand-side of Eq. (6) is an initial time lag at the location $A$ or $B$, and $\Delta t$ in the left is the evolved time lag measured at the other side. The values of $P$ and $Q$ are to be determined in the limit of the small initial $\Delta t$.

The numerical investigations for the coupled MVP model show that $P$ is insensitive to $\mu$ and approximately the unit. Meanwhile, $Q$ strongly depends on $\mu$. The variation of $Q$ versus $\mu$ is shown in Fig. VI for different coupling strengths and different coupling variables as well. As can been seen in the figure the position-coupling (curves 1 and 2) leads to stronger dephasing as $\mu$ approaches the value of the homoclinic bifurcation, $\mu \approx 1.255$. The other case of the velocity-coupling (curves 3 and 4) leads to inphasing. The trend of both inphasing and dephasing becomes stronger as the coupling strength becomes stronger: compare the curves 2,4 for $\varepsilon=0.01$ with the curves 1,3 for the $\varepsilon=0.001$.

\section{VECTOR COUPLING}

For a diffusively coupled system, the coupling term in general would be proportional to the differencies $x_{1}-x_{2}$ and $y_{1}-y_{2}$. A simpler case might be conceived in which the coupling is through only a single variable, either the position or the velocity. For instance, the position-coupling has been often considered in the studies of the coupled oscillators. The examples include the electronic circuits with a purely resistive coupling between component circuits, the inertial coupling for mechanical oscillator systems, and the neuron models with the electric coupling. In more realistic circumstances, however, the two-variable coupling seems to be more natural. For instance, the reactance present in electronic circuits or the propagation time delay of the impulses along the neuronal axon may well require couplings between oscillator units through the velocity variable.

In the followings we attempt to consider the general case of the two-variable coupling and choose a form called the vector coupling. That is, the coupling is introduced using a vector $\mathbf{K}=\left(K_{x}, K_{y}\right)$ as follows:

$$
\begin{aligned}
& \dot{x_{1}}=y_{1}+K_{x}\left(x_{2}-x_{1}\right) \\
& \dot{y_{1}}=-F_{1} y_{1}-F_{2}+K_{y}\left(y_{2}-y_{1}\right)
\end{aligned}
$$

For the other oscillator the coupling term is obtained by interchanging $x_{1}$ and $y_{1}$ with $x_{2}$ and $y_{2}$, respectively. That is, the coupling is given symmetrically. Such form of coupling has been previously considered for studies in different contexts $[7,8]$.

In the present work we represent the vector coupling using the polar coordinate:

$$
\begin{aligned}
& K_{x}=K \cos \Psi, \\
& K_{y}=K \sin \Psi .
\end{aligned}
$$

That is, $K$ denotes the coupling strength and the angle $\Psi$ denotes the relative weight of coupling between two variables. $\Psi$ can be also viewed as the orientation angle of the coupling force in the two-dimensional subspace of each oscillator. A special case occurs when $\Psi=\pi / 4,5 \pi / 4$ in which the 
coupling force on an oscillator is directed toward the other. The single-variable coupling cases are achieved when $\Psi=0, \pi$ (the position-coupling) and $\Psi= \pm \pi / 2$ (the velocity-coupling), respectively. The 'purely' diffusive coupling refers to the regime where neither $K_{x}$ nor $K_{y}$ is negative, that is, where $0 \leq \Psi \leq \pi / 2$.

\section{SYNCHRONIZATION OF THE COUPLED MVP OSCILLATORS}

The presentation of the observations on the synchronization behaviors of the coupled MVP equations are divided in three subsections below. The first part considers the case when the coupling strength is sufficiently weak so that an analytic method can be applicable. The second part considers the case of the finite coupling strength and shows how the results in the weak coupling limit extends in this regime, let alone some additional behaviors. The third part considers the stronger coupling strength regime where the coupled oscillators are placed in an intermediate distance to the homoclinic bifurcation.

\section{A. Weak coupling limit}

Firstly, we consider the weak coupling case in which the coupling raises only a negligible perturbation to the limit cycles of the uncoupled oscillators. It is well known that such limits can be analyzed using the phase model reduction method [1,14]. That is, in this scheme, each limit cycle is approximated with the uncoupled one and the phase dynamics between oscillators due to coupling can be analyzed merely from the antisymmetric part, $\Gamma_{a}^{e f f}(\Delta \phi)$, of the effective coupling function defined as

$$
\Gamma^{e f f}(\Delta \phi)=\frac{1}{2 \pi} \int_{0}^{2 \pi} Z(\phi) p(\phi, \Delta \phi) d \phi
$$

where $\Delta \phi$ denotes the phase difference between two oscillators and $p(\phi, \Delta \phi)$ denotes the perturbation due to coupling that depends on the oscillator phases. The sensitivity function, $\left.Z(\phi) \equiv \nabla_{X} \phi\right|_{X=X_{0}(\phi)}$, measures the phase-dependent reponse of the uncoupled limit cycle $\left(X_{0}\right)$ to the perturbation.

Then, the zeroes of $\Gamma_{a}^{e f f}(\Delta \phi)$ correspond to the phase-locked synchronization states and their stabilities are determined from the slope of $\Gamma_{a}^{e f f}(\Delta \phi)$ at the corresponding states: the negative slope means a stable state, and vice versa. Some typical behaviors of $\Gamma_{a}^{e f f}(\Delta \phi)$ at different parameter values are shown in Fig. VI. The three curves in the figure correspond to the three main kinds of the synchronized states: the inphase $(I)$, antiphase $(A)$, and out-of-phase synchronization $(O)$. Due to the symmetry of Eq. (7), the existence of the inphase state is trivial. The existence of the antiphase state is also guaranteed due to the periodicity of $\Gamma_{a}^{e f f}(\Delta \phi)$. The out-of-phase state corresponds to the phase-locked state with phase difference between zero and $\pi$. The symmetry of Eq. (7) is broken for the out-of-phase states (also for $A$ ), but they occur as a pair each of which is symmetric to the other.

From the phase model analysis we observe that different states of synchronization exist depending on the paramemter values of $\mu$ and $\Psi$ and the parameter space is basically divided into four different regions. Fig. VI(a) shows the phase diagram in the polar coordinated plane of $(\mu, \Psi)$. The range of 
$\mu$ is given such that the radius of the plane is limited to the $\mu$ value for the homoclinic bifurcation. In the figure the blank area corresponds to the inphase synchronization states $(I)$, the dark grey area to the antiphase states $(A)$, and the dashed area to the out-of-phase states $(O)$. The overlap of the $I$ and $A$ areas is denoted by the light grey area $(C)$ in which the inphase states and the antiphase states coexist. The scale of the radial axis has been nonlinearly transformed to magnify the behavior at larger $\mu$ values.

For smaller $\mu$ values $(\mu<\sim 0.5$, roughly), the diagram shows that the synchronization behavior is qualitatively equivalent to that of the coupled van der Pol oscillators. Namely, the inphase synchronization is the only stable state in the (purely) diffusive coupling regime. The synchronization states are either of inphase $(I)$ or of antiphase $(A)$, depending on the coupling angle $\Psi$; the antiphase state is stable only when the coupling $\left(K_{x}, K_{y}\right)$ is negative, in which case the coupling is repulsive rather than being diffusive (attractive).

For larger $\mu$ values, however, synchronization depends not only on $\Psi$ but also on $\mu$, and one more kind of synchronization exists, that is, the out-of-phase state $(O)$. To see the parameter dependence of the behavior, let us set $\mu=1.2$ and, starting from some value within the $I$ region, say $\Psi=3 \pi / 4$, increase $\Psi$ along the circular path as denoted in Fig. VI(a). The inphase state is the only stable state until it reaches $P_{1}$ where the Floquet multiplier of maximum magnitude becomes +1 . The inphase state loses stability at this point and two other stable states with the broken symmetry ( $O$ states) are born. The curve of the symmetry-breaking bifurcations is denoted as $S B_{1}$ in the figure. As $\Psi$ is increased, the pair of the out-of-phase states collide to each other and disappear at $P_{2}$ where the inverse symmetry-breaking bifurcation $\left(S B_{2}\right)$ occurs, which in turn gives birth to a stable antiphase state $(A)$.

When $\Psi$ is further increased the inphase state becomes stable at $P_{3}$, while the antiphase state still remains stable. That is, there exists a region where both the inphase and the antiphase state are stable, as denoted by $C$ in the figure. They coexist until the antiphase state loses its stability at $P_{4}$ upon the symmetry-breaking bifurcation with increased $\Psi$. The bifurcation curves passing through $P_{3}$ and $P_{4}$ are denoted in Fig. VI(a) as $S B_{3}$ and $S B_{4}$, respectively. The bifurcations at $S B_{3}$ and $S B_{4}$ are subcritical in that they are entailed by the presence of two unstable (out-of-phase) states.

To summarize the contrasted behaviors, the phase diagram of Fig. VI(a) shows that the inphase synchronization is the only stable state for the weak diffusive coupling $(0 \leq \Psi \leq \pi / 2)$, similar to the behaviors of the coupled van der Pol oscillators, only when $\mu$ is sufficiently small, that is, when the limit cycle is far from the homoclinic bifurcation. However, it also shows that the inphase synchronization may not be the only stable state even in the regime of the diffusive coupling, when the limit cycle approaches the homoclinic bifurcation with increased $\mu$. Such tendancy seems to be more outstanding for the case of the position-coupling especially, as one can notice from the presence of the antiphase synchronization as the only stable state in the regime of $\Psi \sim 0$.

The three synchronization states occur by exchanging their stabilities under the symmetry-breaking bifurcations. The occurrence of the symmetry-breaking bifurcations along the circular path of 
Fig. VI(a) is schematically depicted in Fig. VI(b). The circles at the four sites represent the variation of the phase difference $\Delta \phi$ and the smaller circles on them denote the synchronization states; the filled small circle denotes the stable state and the empty circle denotes the unstable state. In the insets of the bifurcation diagrams the branches for inphase and antiphase states are denoted as straight lines and the emerging pairs of branches for symmetry-breaking $O$ states are denoted as parabolic curves. A solid line denotes a stable branch and a dotted line an unstable branch.

\section{B. Finite coupling strength}

When the coupling strength becomes finite, the perturbation of the limit cycle due to coupling can be significant and, consequently, the phase model reduction may not be appropriate for predicting the behavior of the coupled dynamics. Then, one needs to resort to direct numerical methods.

In this subsection, using the techniques of the bifurcation analysis, we examine the synchronization behaviors of the coupled MVP model over a range of the coupling strength, focusing on how the results of the weak coupling limit in the previous subsection extend in the regime of the finite coupling strength. Also we obtain the phase diagram for the coupled Morris-Lecar model and compare it with the one for the MVP model to show that the proposed model in a moderate range of the parameters may well display the generical behaviors of the coupled oscillator systems near the homoclinic bifurcation.

Since our primary interest is on the limit cycle oscillations near the homoclinic bifurcation, we fix $\mu=1.2$ for the both oscillators, which is close to the bifurcation point, and then vary the two coupling parameters, $K$ and $\Psi$. In particular, our view of interest is limited to the region of the phase space where each oscillator is in a stable oscillatory state; we may call this region as 'the region of coupled oscillations'. Therefore, whenever trajectories leave the region via a boundary crisis, we assume that there are no stable attractors in the region. The detailed mechanism for the crisis is beyond the present scope.

The resulting phase diagram in the polar coordinated plane $(K, \Psi)$ is shown in Fig. VI(a) in the regime of $K<\sim 0.01$. The occurrence of the boundary crisis is denoted as $B C$ and the region of the parameter space with no attractors in the region of coupled oscillations is colored black in the diagram. Notice that Fig. VI(a) and Fig. VI(a) are drawn in the different parameter planes.

As shown in the weak coupling limit, there exist three main kinds of the synchronization states: the inphase $(I)$, antiphase $(A)$, and out-of-phase $(O)$ states. As $K$ becomes finite, the region of each state starts to be deformed from the phase model prediction, which is manifested by the deflection of the bifurcation lines that depends of $K$. Besides the symmetry-breaking bifurcation described previously, the states may also undergo other bifurcations such as the period-doubling that cannot be predicted from the phase model description either.

Typical behaviors of the transitions and their coexistences are depicted in Fig VI(b) along the $\Psi$ paths with two different $K$ values: the two paths are labeled as shown in Fig.VI(a). The branches 
in the diagrams are drawn using the same convention as in Fig. VI(b). In each subset the upper horizontal line denotes the inphase state branch $(I)$ and the lower line denotes the antiphase state branch $(A)$. Note that the diagram depicted for the path 1 of smaller K, coincides with the behavior observed in the case of the weak coupling limit (Fig. VI(b)).

For the path 2 of larger $K$, we observe the new behavior of the period-doubling cascades of the out-ofphase states. The cascades at the symmetric branches lead to the onset of chaos which are symmetric to each other as well. As $\Psi$ further decreased, two chaotic attractors merge to form a single chaotic attractor, which then restores the symmetry. This symmetric chaotic attractor eventually disappears via a boundary crisis and the trajectory leaves the region of coupled oscillations. The period-doubling cascades of the out-of-phase states also occur at different regimes of stronger coupling strength.

In Fig. VI(b), from the diagram corresponding to the path 2, one may also notice that the saddlenode bifurcation of the out-of-phase states provides an additional source for the birth of a pair of the $O$ states, which can be viewed as the connection of a stable and an unstable branch of the $O$ state that originate from different states, $I$ and $A$, respectively. This saddle-node bifurcation is not observed in the weak coupling limit (Fig. VI(a)) even though they can occur in the phase model description; the saddle-node bifurcation occurs at the tangency of $\Gamma_{a}^{e f f}$ in Fig. VI. The region of these $O$ states is denoted in Fig. VI(a) as the dark dashed area.

For a comparison with the neuronal model, the phase diagram for the coupled Morris-Lecar model is shown in Fig. VI. For this model the homoclinic bifurcation takes place at $I_{d c} \approx 0.0729$ and the diagram has been obtained at $I_{d c}=0.0750$, close to the homoclinic bifurcation. The broader region of chaos following the period-doubling cascades is denoted by the hatched area in the figure. A peculiar cusp point $(C P)$ appears to which many regions for different states merge, which is commented briefly in the following subsection.

The organization of the various states for the Morris-Lecar model is not precisely the same as for the MVP model, which should vary depending on specific models. It is likely that the range of the parameters such as $I_{d c}$ and $K$ has not been chosen to the best to show a better coincidence. Nevertheless, the features in the regime of the weak coupling strength are essentially the same for the both models in terms of the existence of the bifurcations and the pattern of their occurrences. More importantly, the diagrams show that the both systems have the stable dephased synchronization in a wide range of the diffusive coupling regime, which has been the primary interest of the present examination.

\section{Intermediate regime of the coupled oscillator}

So far we have emphasized a contrast between the behaviors of the coupled oscillators in two distinctive regimes in the aspect of the closeness to the homoclinic bifurcation. That is, the coupled van der Pol-type oscillators and the coupled oscillators near the homoclinic bifurcation. In this subsection we examine the behavior of the coupled MVP model in an intermediate regime between 
those distinctive regimes, which we may consider to be provided by setting the parameter at $\mu=1.0$. In particular, the case of the stronger coupling strength is considered since it turns out that the weak coupling results in rather trivial behaviors that have been already observed from those two regimes. The phase diagram in this regime of the parameters is shown in Fig. VI which demonstrates a variety of complex behaviors.

The inphase synchronization states, in addition to the symmetry-breaking bifurcation described previously, may undergo two more kinds of bifurcations: the period-doubling and the torus bifurcation. The period-doubling either gives birth to a stable period-doubled inphase state, or to none of stable attractors. In the former case the period-doubled inphase state undergoes the symmetry-breaking bifurcation, which in turn gives birth to a pair of the out-of-phase states. These out-of-phase states undergo the cascade of period-doublings leading to chaos in the same way as observed for the $O$ states in Fig. VI. Two such cases are shown in Fig. VI to the northwest and the southeast directions.

The latter case of the period-doubling bifurcation entailing no attractors implies the occurrence of the boundary crisis with an attractor outside the region of coupled oscillations. Therefore, the trajectory suddenly disappears from the region of coupled oscillation. The regime of the paprameters of this case is denoted by the black area in the diagram. The period-doubling bifurcations of the inphase states resulting in these two cases also occur for the Morris-Lecar model as can be found in Fig. VI.

The torus bifurcation of the inphase states occurs when a complex conjugate pair of the Floquet multipliers leaves the unit circle in the complex plane. The bifurcating torus is observed to retain the symmetry of Eq. (7). The flows on the torus are just the ones for the coupled systems with two competing frequencies. Below the curve for the torus bifurcation $(T)$ in Fig. VI(a), note the existence of the familiar resonant tongues corresponding to the frequency-locked states with rational rotation numbers. The most prominent among them is the tongue of the one-to-one locking, which is denoted in the diagram. Notice also that the tongues can persist even in the absence of the stable torus nearby in the parameter space. The torus disappears via a boundary crisis. The torus bifurcation also occurs for the Morris-Lecar model even though such case is not indicated in Fig. VI; it is observed to occur at a stronger coupling strength, $K \sim 0.15$.

A special notice is on the existence of the cusp points in the northwest and the southeast directions in Fig. VI ( $C P$ in the diagram), to which all the regions of the period-doubling merge to form a common boundary; they are observed to be the cusp points within the numerical resolution. Such location implies that the codimension of the bifurcation would be infinite. Interestingly, the cusp point also exists in the Morris-Lecar model as shown in Fig. VI. Possible questions such as its genericity and the unfolding prompt further investigations.

Again, the global organization pattern of the phase diagram in fig. VI is not quite the same as for the Morris-Lecar model in Fig. VI. However, as we have already noticed in this subsection the coincidences between the local behaviors in the two models are striking. Therefore, in this sense of similarities, it seems that the proposed MVP model represents well the Morris-Lecar model over a 
wider range of the parameter space not just restricted to the neighbors of the homoclinic bifurcation with a rather weak coupling strength. We presume that this representation can be also approriate for other models having the structure of Fig. VI.

\section{CONCLUSIONS}

Synchronization between coupled oscillations has been shown to display distinctive behaviors as the limit cycle oscillation approaches the homoclinic bifurcation. In this paper, a generic physical model for studying such behaviors has been proposed using the modified van der Pol equation. A general form of coupling has been also considered by introducing the vector coupling between the variables of the two-dimensional oscillators.

The homoclinic bifurcation implies the presence of a saddle nearby the limit cycle. The dephasing mechanism of the synchronized oscillations in the vicinity of the saddle has been analyzed both qualitatively and quantitatively in the weak coupling limit. The dephasing rate measured by the linear rate $Q$ is shown to increase dramatically as the limit cycle approaches the homoclinic bifurcation and such tendancy becomes even enhanced as the coupling strength is enlarged.

The synchronization behaviors of the coupled MVP oscillators have been examined over a wide range of the coupling parameters. In the weak coupling limit the phase model reduction method has been used to show the existence of the main synchronization states and to identify the transitions among them through the symmetry-breaking bifurcations. For the finite coupling strength we have resorted to the direct numerical calculations using the techniques of the bifurcation analysis, which has revealed the extended behaviors that cannot be predictable from the phase model description. In both cases the phase diagrams have been obtained and it has been shown that the inphase synchronization may not be the only stable state in the regime of the diffusive coupling as the limit cycle approaches the homoclinic bifurcation.

The intermediate regime has been also examined where the coupled oscillators are in between the two distinctive regimes of the van der Pol-type oscillators and the oscillators near the homoclinic bifurcation. A variety of complex behaviors, including the period-doubling and the torus bifurcations, the mode-locking tongues and chaos arises in this regime as the coupling strength becomes larger, for which the phase diagram has been also constructed.

The phase diagrams for the MVP model has been compared with the one for the the Morris-Lecar model which is only an example of the neuronal models that have provided motivations to the present study. The comparison leads to a reasonable conclusion that the synchronization behaviors observed in the MVP model should be generic for the systems of coupled oscillators near the homoclinic bifurcation. The close coincidences between the local behaviors of the models have been also observed over a wider range of the parameter space which is not restricted only to the neighbors of the homoclinic bifurcation with a rather weak coupling strength. 


\section{ACKNOWLEDGMENTS}

The authors would like to thank Y. Kuramoto, C. Kurrer, S. Kim, and A. Neiman for useful discussions. D. P. was supported by the STEPI of Korea through the Korea-Russia scientist exchange program during his stay at the Chungbuk National university. S. K. H. was supported by the BSRC27-2436 and the interdisciplinary research project of the Ministry of Education. H. K. is thankful for the support of the Abdus Salam ICTP for his visit during which the part of the present work was done. This work was done within the framework of the Associateship Scheme of the Abdus Salam International Centre for Theoretical Physics, Trieste, Italy.

\section{References}

[1] Y. Kuramoto, Chemical Oscillations, Waves, and Turbulence, (Springer-Verlag, Tokyo, 1984).

[2] A. T. Winfree, The Geometry of Biological Time (Springer, New York, 1980).

[3] C. M. Gray, P. König, A. K. Engel, and W. Singer, Nature 338, 334 (1989).

[4] R. Eckhorn, R. Bauer, W. Jordan, M. Brosch, W. Kruse, M. Munk, and H. J. Reitboeck, "Coherent oscillations: A mechanism of feature linking in the visual cortex?", Biol. Cybern. 60 121-130 (1988).

[5] C. von der Malsburg and C. Schneider, 'A neural cocktail-party processor', Biol. Cybern. 54, 29-40 (1986).

[6] J. Guckenheimer and P. Holmes, Nonlinear Oscillations, Dynamical Systems, and Bifurcations of Vector Fields, (Springer-Verlag, New York, 1983).

[7] R.H. Rand and P.J Holmes, 'Bifurcation of periodic motions in two weakly coupled van der Pol oscillators', Int. J. Nonlin. Mech., 15, 387 (1980).

[8] D.G. Aronson, E.J. Doedel, and H.G. Othmer, 'An analytical and numerical study of the bifurcation in a system of linearly-coupled ocillators', Physica 25D, 20 (1987).

[9] A. Sherman, J. Rinzel, 'Rhythmogenic effects of weak electronic coupling in neuronal models", Proc. Nat. Acad. Sci. (USA) 89, 2471-2474 (1992).

[10] S. K. Han, C. Kurrer, and Y. Kuramoto, 'Dephasing and Bursting in Coupled Neural Oscillators", Phys. Rev. Lett. 75, 3190-3193 (1995).

[11] A. L. Hodgkin, and A. F. Huxley, 'A Quantitative Description of Membrane Current and its Application to Conduction and Excitation in Nerve", J. Physiol. London 117, 500-544 (1952).

[12] C. Morris, and H. Lecar, "Voltage oscillations in the barnacle giant muscle fiber", Biophys. J. 35, 193-213 (1981).

[13] J. Hindmarsh and M. Rose, A Model of Neuronal Bursting using Three Coupled First Order Differential Equations, Proc. R. Soc. London, B221, 87-102 (1984).

[14] D. Hansel, G. Mato, \& C. Meunier, "Phase dynamics for weakly coupled Hodgkin-Huxley neurons," Europhys. Lett.,23(5), 367-372 (1993). 


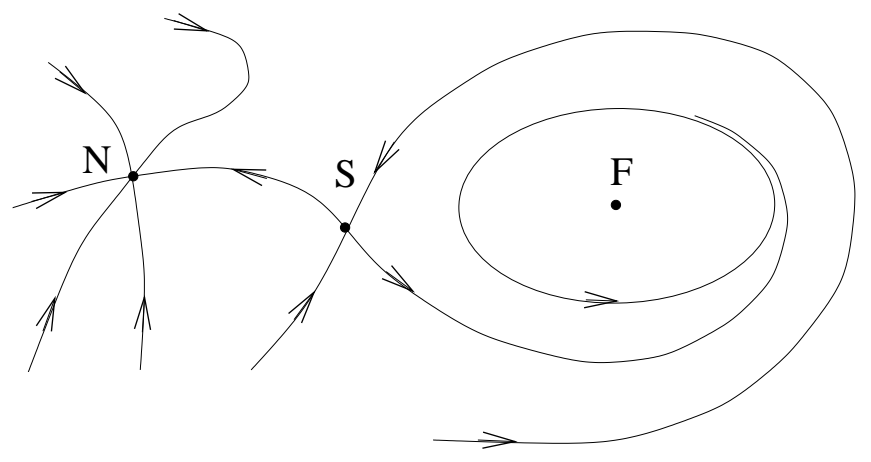

FIG. 1. Typical phase portrait of the neuronal oscillators.
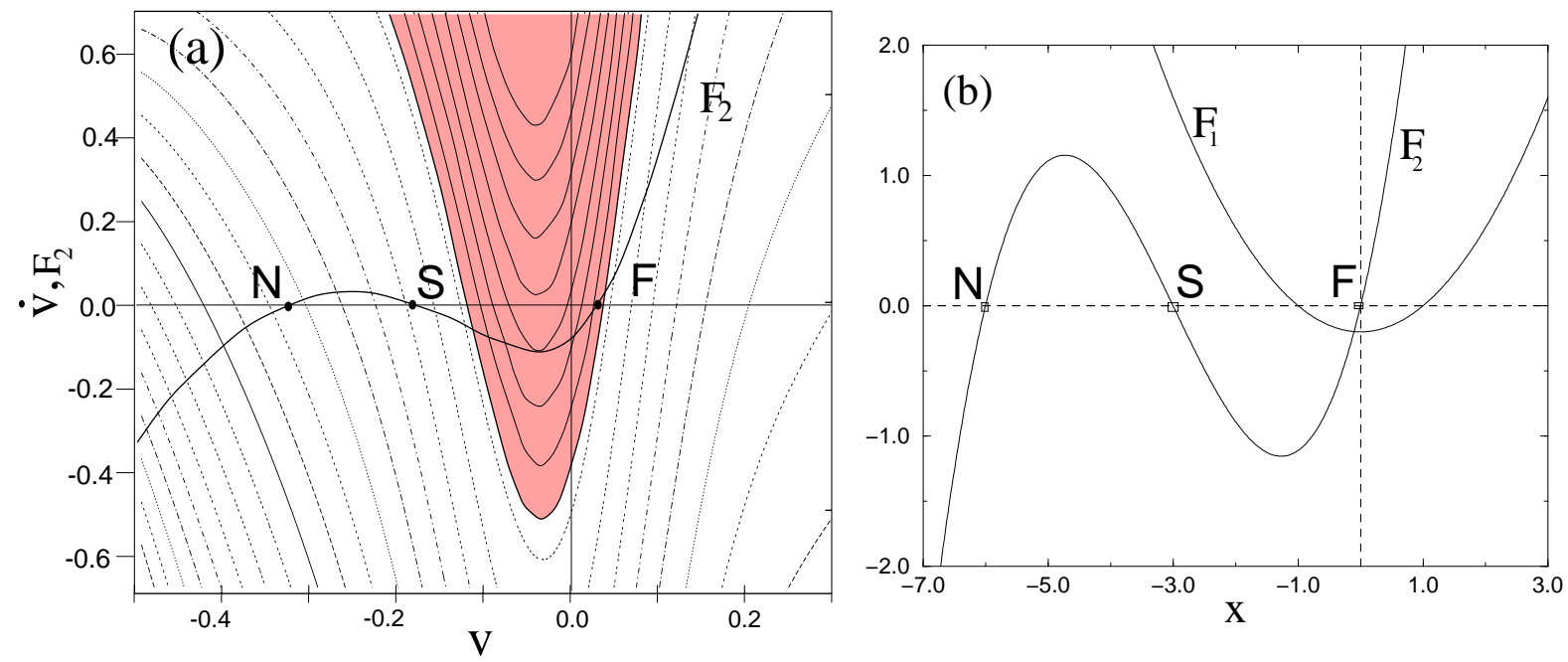

FIG. 2. (a) Function plot of $F_{2}(v)$ and contour plot of $F_{1}(v, \dot{v})$ for the Morris-Lecar model. The dark area corresponds to the negative dissipation, i. e., $F_{1}<0$. (b) Function plots of $F_{1}(x)$ and $F_{2}(x)$ for the MVP model.

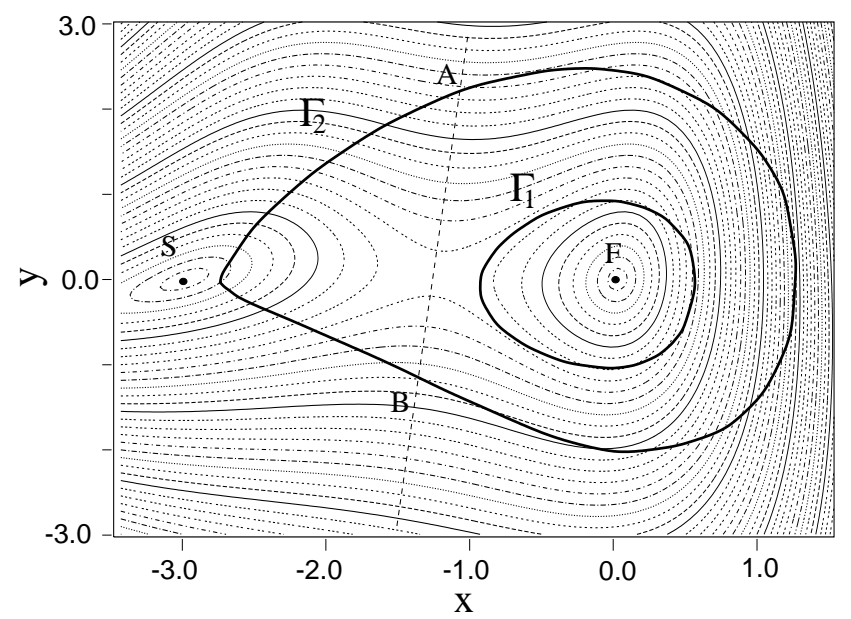

FIG. 3. Contour plot of $v_{\phi}$ and location of limit cycles for the MVP model. The limit cycles are drawn for $\mu=0.2\left(\Gamma_{1}\right)$ and $\mu=1.0\left(\Gamma_{2}\right)$. 


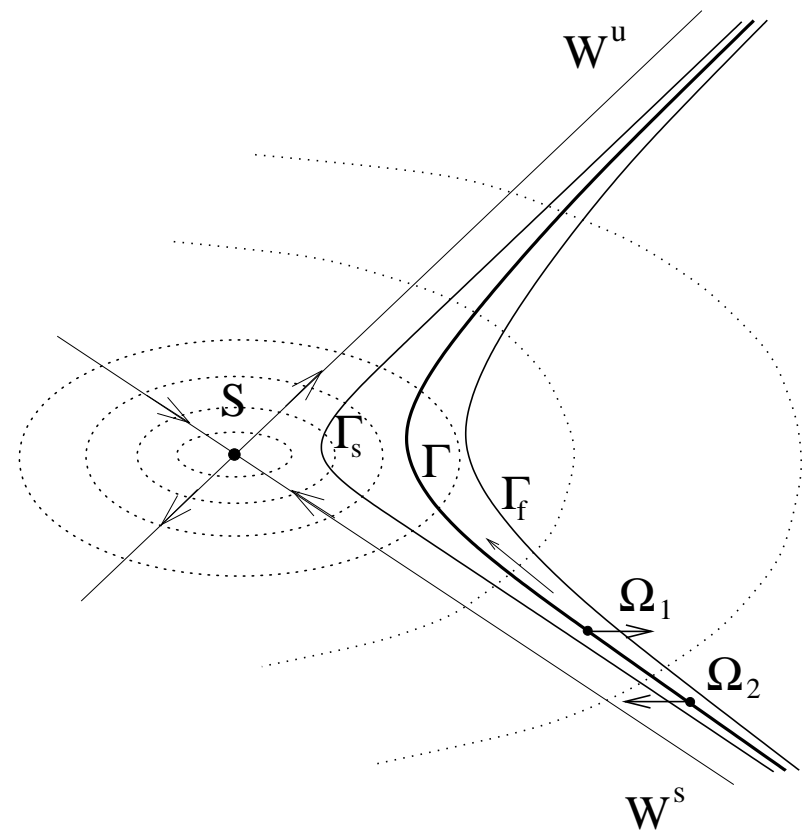

FIG. 4. Phase flows near the saddle $(S)$.

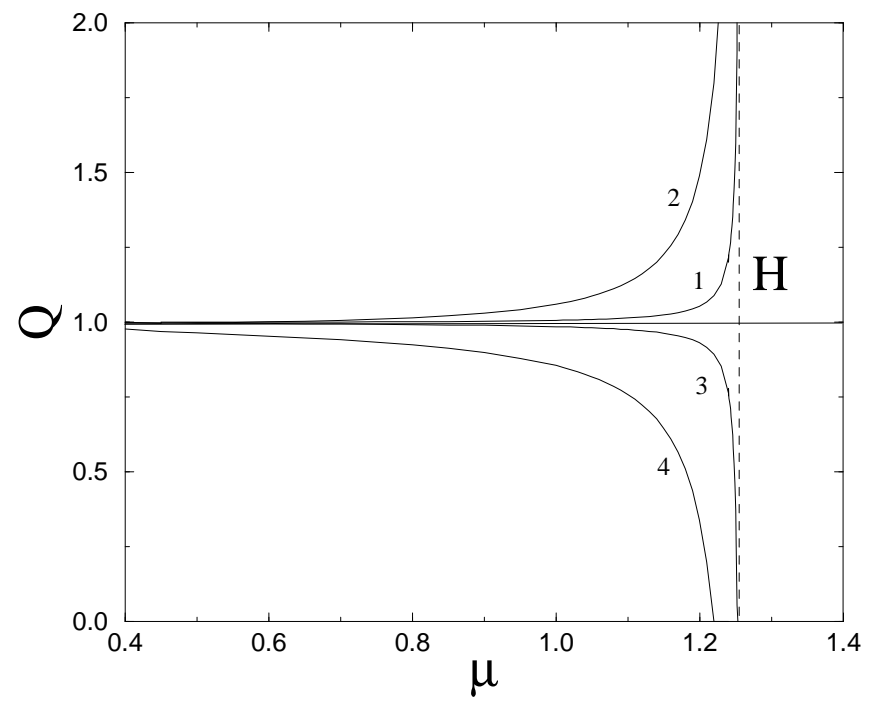

FIG. 5. $Q$ versus $\mu$, the linear dephasing rate. The dotted line $H$ denotes the homoclinic bifurcation point. 


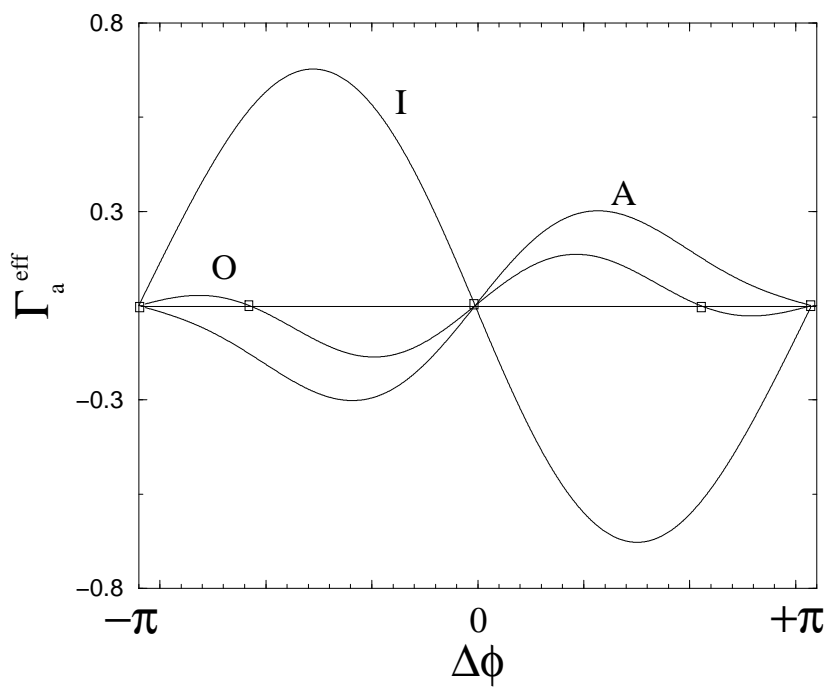

FIG. 6. Plots of $\Gamma_{a}^{e f f}(\Delta \phi)$ at three different parameter values. The label for each curve denotes the corresponding stable synchronization state and the locations of the states are marked by the small squares.
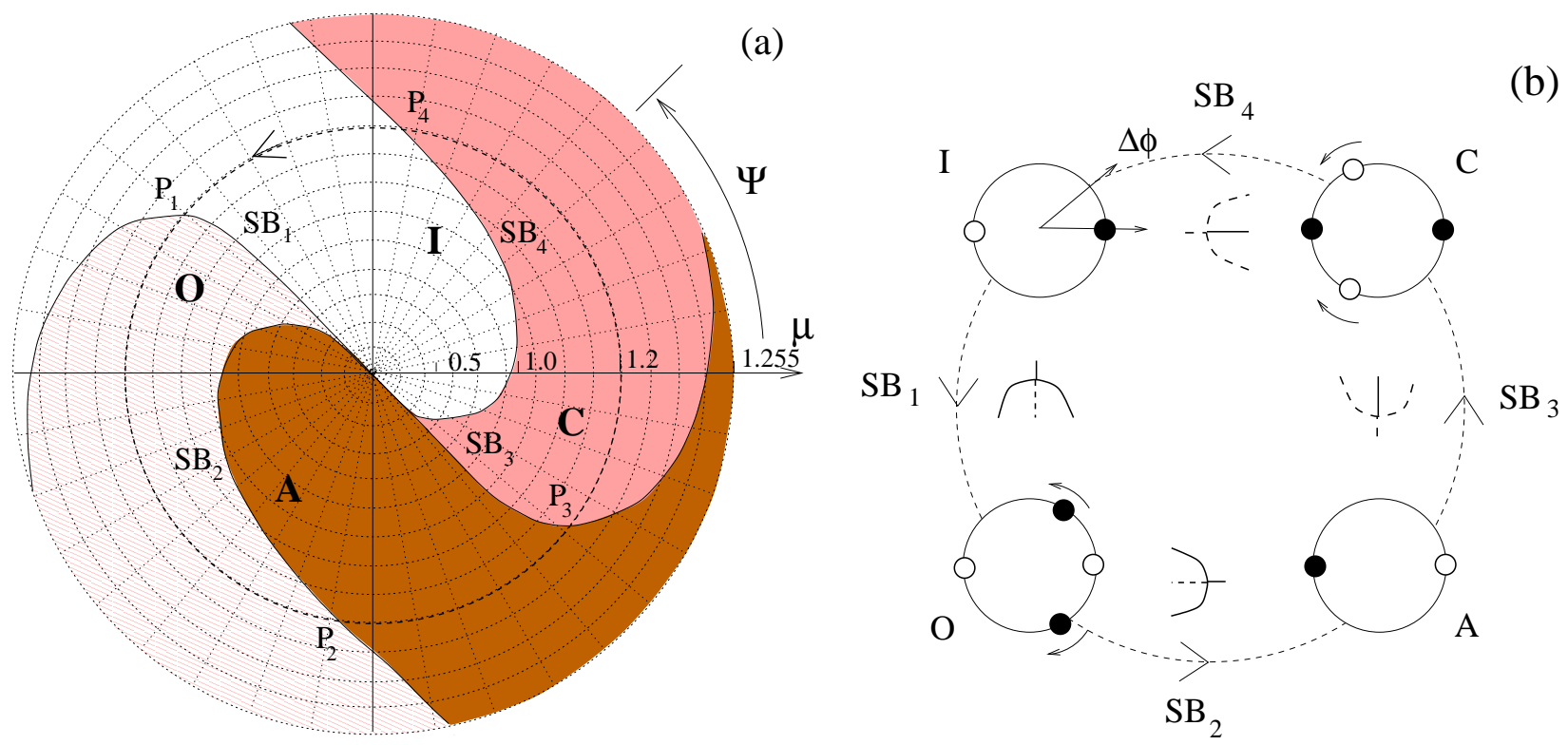

FIG. 7. (a) Phase diagram for the coupled MVP model in the weak coupling limit. (b) Stability and bifurcation of the synchronization states along the circular path of (a). The insets denote the corresponding symmetry-breaking bifurcations among them. 
(a)
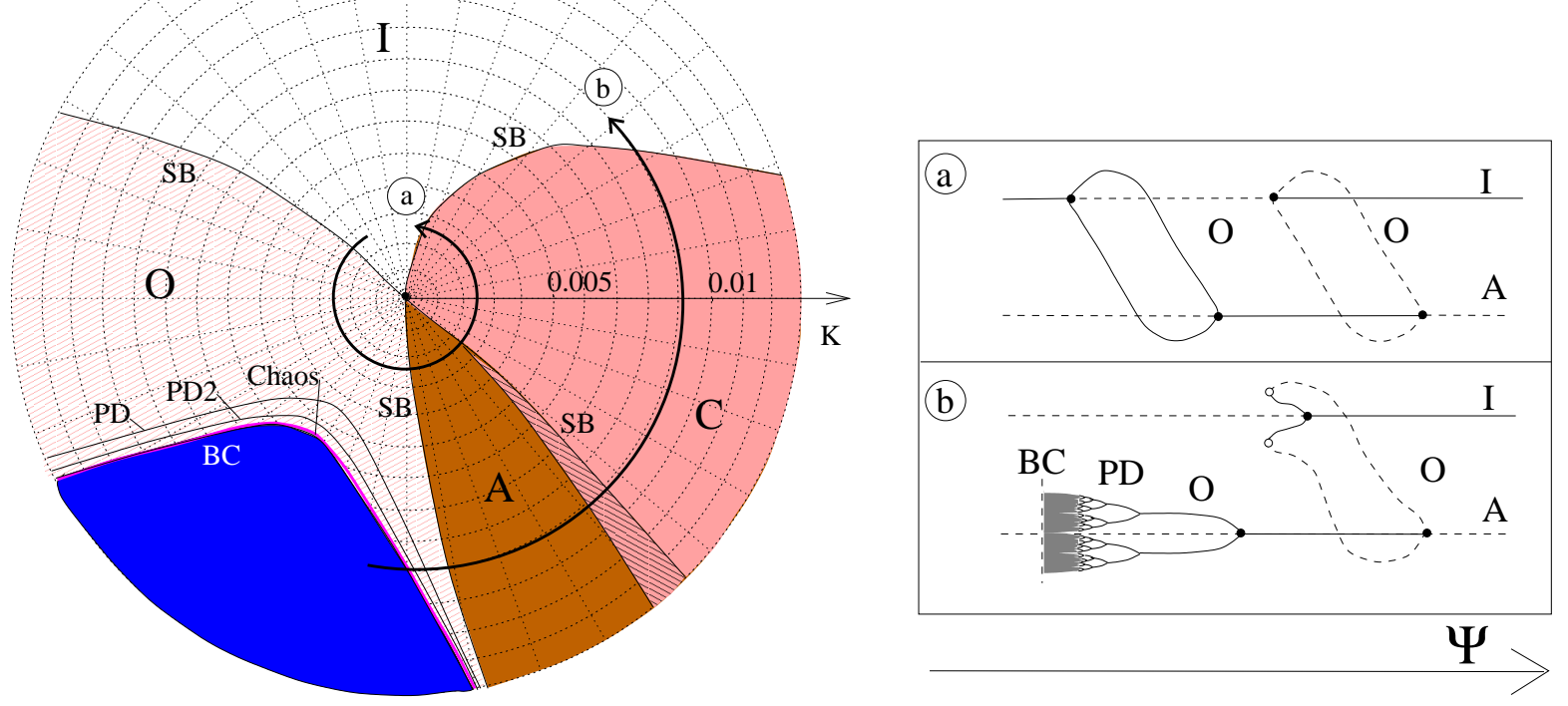

FIG. 8. (a) Phase diagram for the coupled MVP model at $\mu=1.2$ upto finite coupling strength. (b) Bifurcation diagrams along the paths denoted in (a).

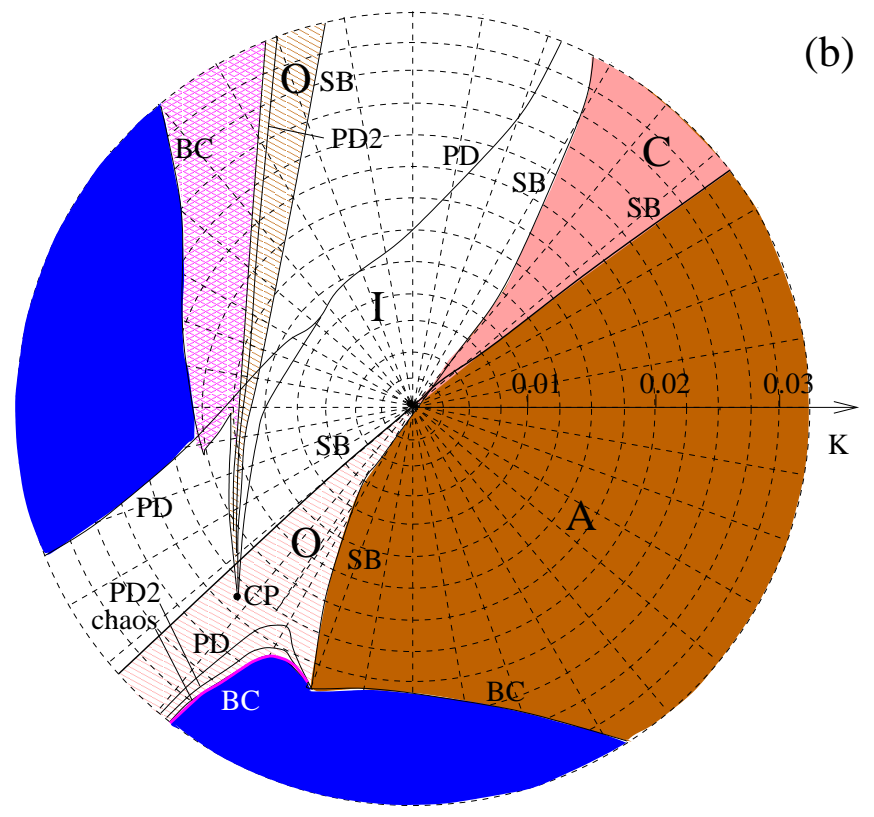

FIG. 9. Phase diagram for the coupled Morris-Lecar model at $I_{d c}=0.0750$. 


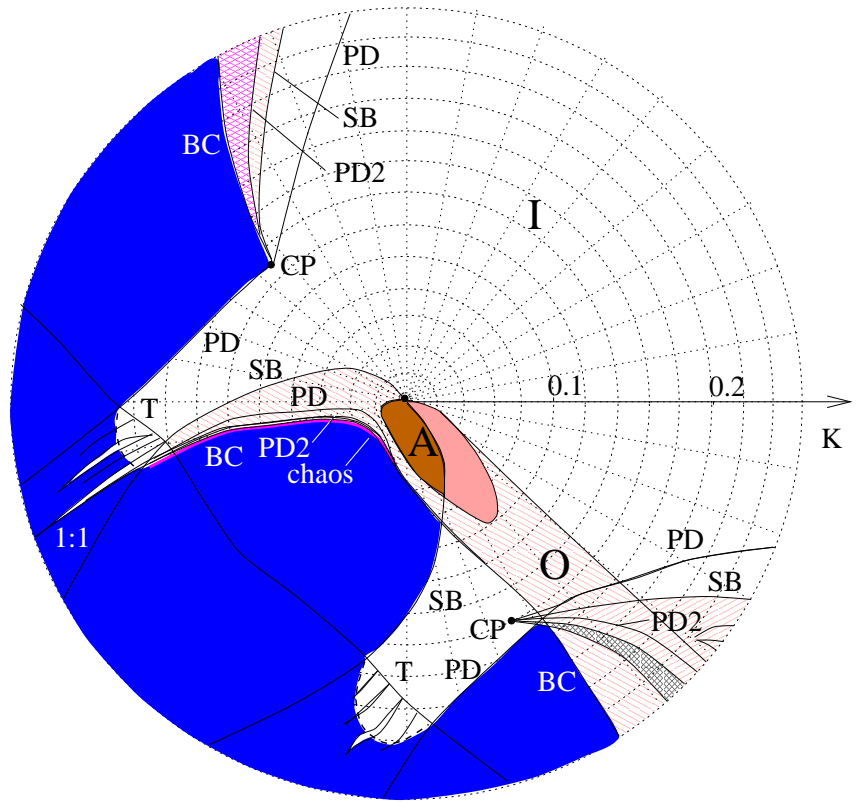

FIG. 10. Phase diagram for the coupled MVP model at $\mu=1.0$ upto stronger coupling strength. 